\title{
Combustion-derived particles inhibit in vitro human lung fibroblast-mediated matrix remodeling
}

\author{
Hannelore Bové ${ }^{1,2^{*}} \mathbb{D}$, Jens Devoght ${ }^{1}$, Leentje Rasking ${ }^{1}$, Martijn Peters ${ }^{3}$, Eli Slenders ${ }^{1}$, Maarten Roeffaers ${ }^{2}$, \\ Alvaro Jorge-Peñas ${ }^{4}$, Hans Van Oosterwyck ${ }^{4,5}$ and Marcel Ameloot ${ }^{1}$
}

\begin{abstract}
Background: The continuously growing human exposure to combustion-derived particles (CDPs) drives in depth investigation of the involved complex toxicological mechanisms of those particles. The current study evaluated the hypothesis that CDPs could affect cell-induced remodeling of the extracellular matrix due to their underlying toxicological mechanisms. The effects of two ultrafine and one fine form of CDPs on human lung fibroblasts (MRC-5 cell line) were investigated, both in 2D cell culture and in 3D collagen type I hydrogels. A multi-parametric analysis was employed.
\end{abstract}

Results: In vitro dynamic 3D analysis of collagen matrices showed that matrix displacement fields induced by human lung fibroblasts are disturbed when exposed to carbonaceous particles, resulting in inhibition of matrix remodeling. In depth analysis using general toxicological assays revealed that a plausible explanation comprises a cascade of numerous detrimental effects evoked by the carbon particles, including oxidative stress, mitochondrial damage and energy storage depletion. Also, ultrafine particles revealed stronger toxicological and inhibitory effects compared to their larger counterparts. The inhibitory effects can be almost fully restored when treating the impaired cells with antioxidants like vitamin C.

Conclusions: The unraveled in vitro pathway, by which ultrafine particles alter the fibroblasts' vital role of matrix remodeling, extends our knowledge about the contribution of these biologically active particles in impaired lung tissue repair mechanisms, and development and exacerbation of chronic lung diseases. The new insights may even pave the way to precautionary actions. The results provide justification for toxicological assessments to include mechanism-linked assays besides the traditional in vitro toxicological screening assays.

Keywords: Combustion-derived particles, In vitro toxicology, Human lung fibroblasts, Hazard assessment, Matrix remodeling inhibition

\section{Background}

Human exposure to combustion-derived carbonaceous particles (CDPs) has increased substantially in recent years. Traffic exhaust, for example, constitutes a major environmental contaminant of diesel soot or black carbon $(\mathrm{BC})$ derived from the incomplete combustion of fuels. Additionally, multiple carbon black (CB) particle

\footnotetext{
*Correspondence: hannelore.bove@uhasselt.be

${ }^{1}$ Biomedical Research Institute, Hasselt University, Agoralaan Building C, Diepenbeek, Belgium

Full list of author information is available at the end of the article
}

types are intentionally engineered via controlled combustion processes for their use in consumer products like printer toner cartridges, car tires and cosmetics. Consequently, hazardous exposure to this type of particles is not limited to occupational settings but also includes daily receptivity of environmental pollutant particulates [1]. While carbon black and black carbon are different particle types, both are classified as CDPs and find commonality in their combustion-based production process, resembling physicochemistry and demonstrated toxicity in various models [1]. 
The respiratory system is a major route of unintentional exposure to aerosolized carbonaceous particles. Once inhaled, CDPs can reach the deepest regions of the respiratory tract depending on their sizes. Especially, fine (diameter $<2.5 \mu \mathrm{m}$ ) and ultrafine particles (diameter $<0.1 \mu \mathrm{m}$ ) tend to deposit in the deeper bronchialalveolar regions of the lungs where they are not rapidly degraded but accumulate and, eventually, may translocate to the blood and other target organs [1-4].

There is increasing evidence that exposure to CDPs can lead to numerous adverse health effects [1]. Moreover, these particles are thought to be more harmful to human health than particulate matter generated by other means [5-7]. Grahame and Schlesinger, for instance, have concluded that $\mathrm{BC}$ particles are the dominant environmental cause of cardiovascular morbidity and mortality [8]. Furthermore, epidemiological and experimental studies have shown that CDPs may attribute to the modulation and aggravation of pulmonary disorders and can even lead to lung cancer $[1,9,10]$. The constant human exposure to CDPs warrants in depth investigation of the involved toxicological mechanisms of these particles inducing the observed illnesses. Both in vitro and in vivo studies have already elucidated that carbonaceous particle exposure can induce cytotoxic injury, impaired redox regulation, inflammation and tissue remodeling $[1,11,12]$. In chronic lung diseases, to which inhalation of CDPs appears to contribute, tissue remodeling has shown to contribute to structural and functional alterations in the lungs. Yet, the exact toxicological mechanisms involved and their interconnections are still not fully unraveled. In a previous study by our research groups, we have shown that the tubulin cytoskeleton of fibroblasts is heavily disturbed after carbonaceous particle exposure [13]. Consequently, we hypothesize that CDPs may impair cell-induced remodeling of the extracellular matrix (ECM) due to their underlying toxicological mechanism.

Hence, the current study was designed to quantitatively evaluate whether CDPs affect the cellular remodeling of the surrounding matrix and to decipher the distinct toxicological mechanism underlying this detrimental effect. The study was performed using lung fibroblasts. Despite the fact that lung epithelial cells and macrophages constitute the first barrier in the lungs, fibroblasts are as critical to evaluate since they are the main connective tissue cell type and maintain the stroma for numerous other cells including alveolar epithelial cells [14]. In damaged lung tissue, fibroblasts are responsible for the main repair mechanisms including building new ECM and contracting the novel matrix to match it with undamaged tissue $[14,15]$. Consequently, it is of critical importance to evaluate their remodeling capacity under CDP exposure. Here, 3D cell-induced displacement microscopy (CDM) is employed to examine the detrimental effects of CDPs on human lung fibroblasts.

\section{Results \\ Cytoskeletal integrity}

The effects of one fine (CCB; diameter $<2.5 \mu \mathrm{m})$ and two ultrafine (ufPL and ufP90; diameters $<0.1 \mu \mathrm{m}$ ) CDP types were tested of which all key physicochemical characteristics are summarized in Additional file 1: Table S1.

When studying the actin cytoskeleton, it can be seen that the filaments are disturbed after an incubation period exceeding $4 \mathrm{~h}$ (Additional file 1: Figure S1). Moreover, no clear stress fibers are observable compared to the control cell. In agreement with our previous report about the disturbance of the tubulin cytoskeleton after CDP exposure [13], the larger, fine CCB particles have a less pronounced effect on the cytoskeleton than the smaller ultrafine ufPL and ufP90 particles (data not shown).

\section{D cell-induced displacements and matrix remodeling}

Representative images showing 3D collagen matrix displacements induced by embedded fibroblasts exposed to plain culture medium (negative control) or three different CDP types are shown in Fig. 1a-d. The images indicate that the displacements induced by control cells are significantly different from cells exposed to ultrafine and fine particles. This visual observation is confirmed by quantitative analysis (Fig. 1e). The median \pm standard deviation (SD) of 3D cell-induced matrix displacements drops from $4 \pm 1 \mu \mathrm{m}$ (negative control) to $0.4 \pm 0.3,0.9 \pm 0.4$ and $2 \pm 0.5 \mu \mathrm{m}$ for the ultrafine (ufPL, ufP90) and fine (CCB) particles, respectively. Also, it is clear that the ultrafine particles are causing more detrimental effects to the cells ability of structuring the ECM than the larger particles.

The findings correspond well with changes observed in the organization of the ECM after fibroblast remodeling as can be seen from the label-free imaging of the fibrils of the collagen type I matrix using second harmonic generation (Fig. 2a-d). Whereas the control cells nicely contract and align the collagen fibrils at their force poles, the fibril distribution is more randomly oriented around cells exposed to CDPs. The matrix distribution was quantified based on fibril orientation histograms with fitted Von Mises function (Additional file 1: Figure S2). The median \pm SD spread of fibril orientation $(\kappa)$ varies from $17 \pm 3$ (negative control) to $0.2 \pm 0.2,0.9 \pm 1.0$ and $5 \pm 8$ for the ultrafine (ufPL, ufP90) and fine $(\mathrm{CCB})$ particles, respectively Fig. 2e). The lower values after incubation with fine but especially ultrafine particulates indicate that the collagen fibrils are randomly oriented compared to the control condition where the fibrils are highly aligned around the cellular force poles.

To examine if inhibition of cell-mediated matrix remodeling results from cytotoxicity induced by the 

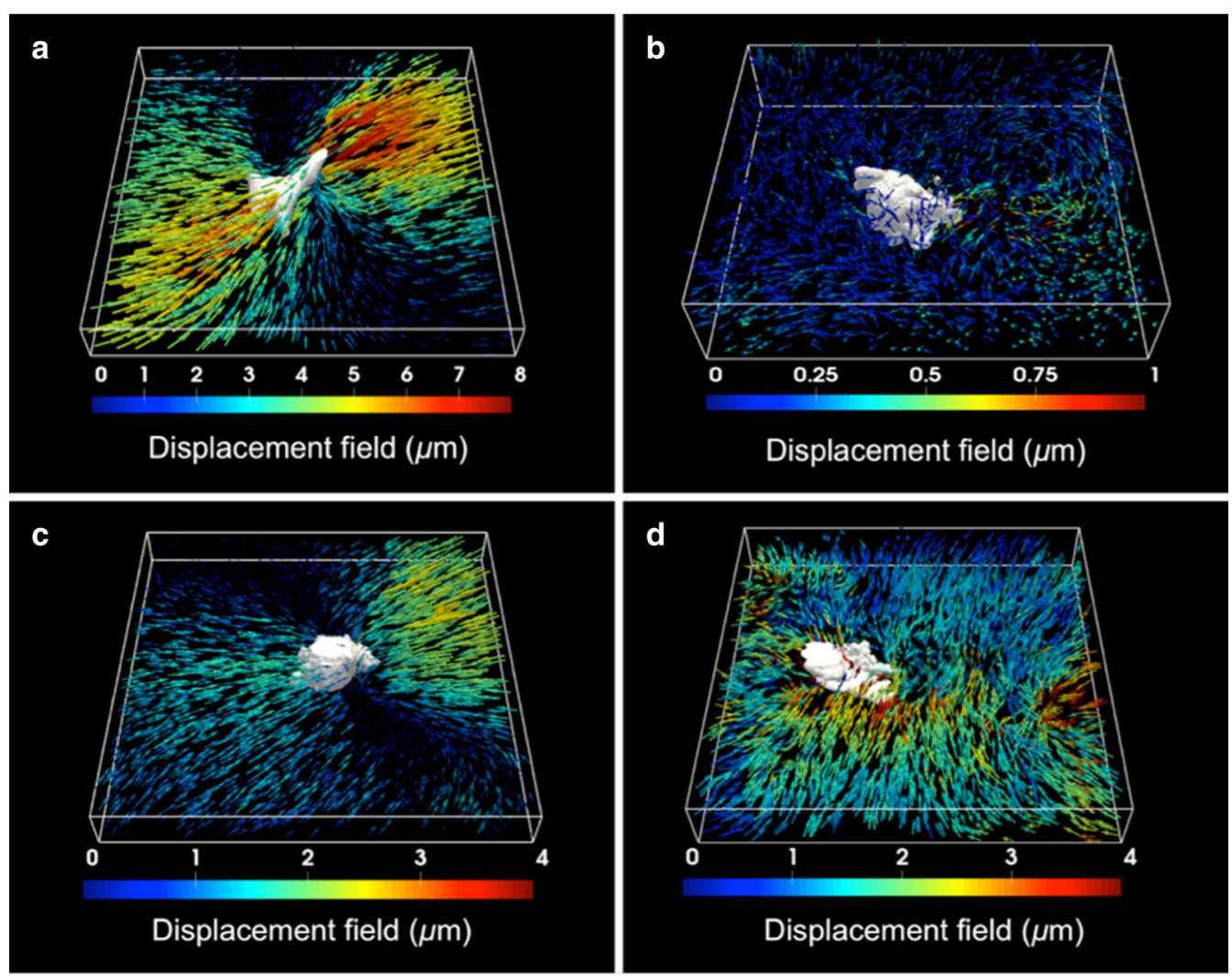

e

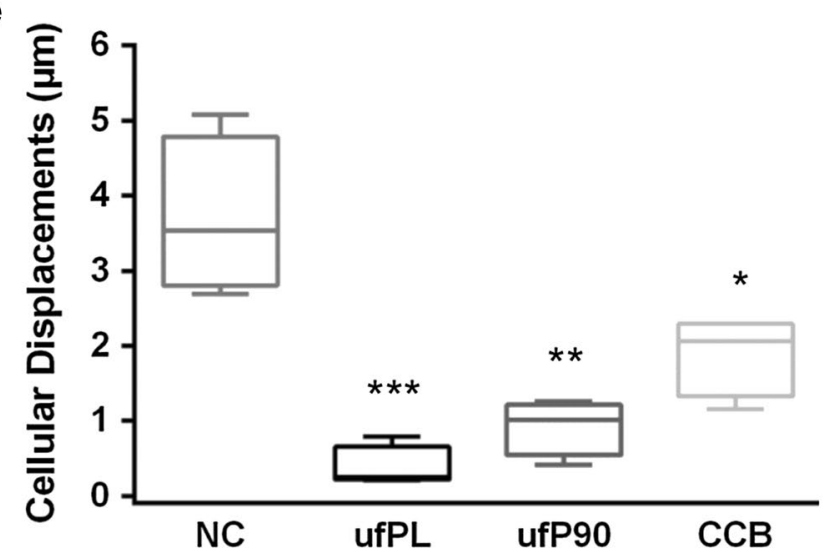

Fig. 1 Impaired human lung fibroblast displacements in 3D collagen type I hydrogels induced by CDPs. Human lung fibroblasts (MRC-5 cell line) were exposed for $24 \mathrm{~h}$ to $20 \mathrm{\mu g} / \mathrm{cm}^{2}$ of three different types of CDPs at $37^{\circ} \mathrm{C}$ and embedded in 3D collagen type I hydrogels to study their ability to generate matrix displacements. Representative 3D displacements induced by fibroblasts (cell body in white) incubated with a culture medium (NC, negative control), bounding box $172 \times 172 \times 21 \mu^{3}$; b ufPL, bounding box $173 \times 172 \times 46 \mu^{3}$; c ufP90, bounding box $167 \times 170 \times 11 \mu m^{3}$; and $\mathbf{d} C C B$, bounding box $172 \times 172 \times 26 \mu^{3}$; obtained from the registration of fibril-based images. Note, on the right some displacements of a lower localized cell are visible. e Data analysis of the 3D distribution of matrix displacements under the different conditions. Data are represented as box-plots (medians and quartiles; $n=4$ ). Statistically different from control ${ }^{*} p<0.005 ;{ }^{* *} p<0.0005 ;{ }^{* *} p<0.0001$

particles, the extent and mode of cell death was investigated. Analysis showed that ultrafine but not fine carbonaceous particles at a concentration of $20 \mu \mathrm{g} / \mathrm{cm}^{2}$ are able to induce significant apoptotic cell death in human lung fibroblasts (Additional file 1: Figure S3).

\section{ROS generation}

To investigate the possibility of a ROS-mediated mechanism responsible for the inhibition of the cellular displacements and resulting impaired matrix remodeling, 

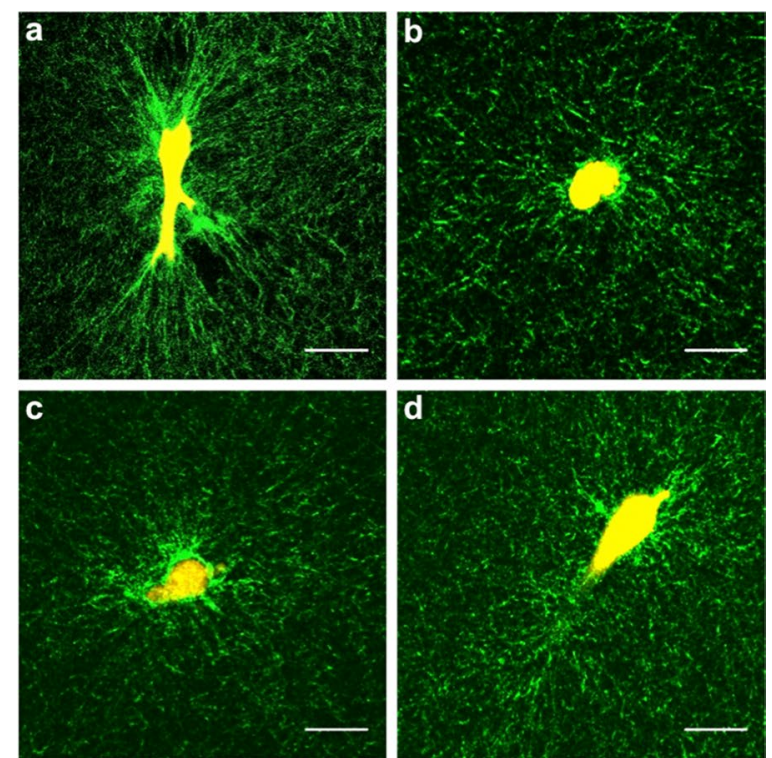

\section{e}

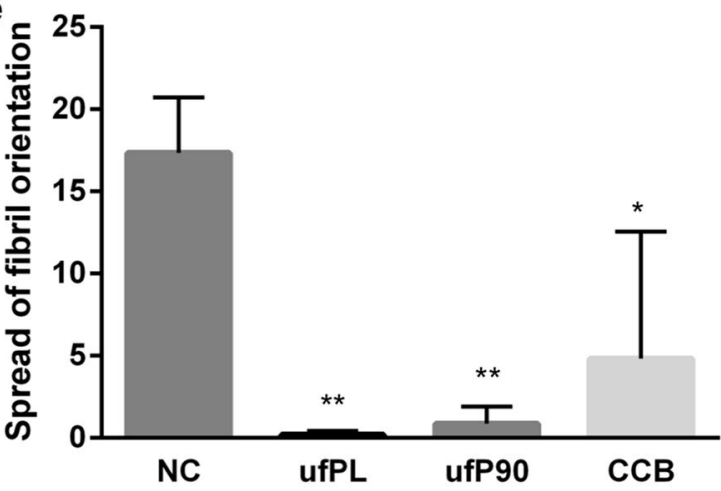

Fig. 2 Inhibited collagen type I matrix remodeling by human lung fibroblasts exposed to CDPs. Human lung fibroblasts (MRC-5 cell line) were exposed for $24 \mathrm{~h}$ to $20 \mathrm{\mu g} / \mathrm{cm}^{2}$ of three different types of CDPs at $37^{\circ} \mathrm{C}$ and embedded in $3 \mathrm{D}$ collagen type I hydrogels to study cell-mediated matrix remodeling. Representative images of collagen type I (second harmonic imaging, green) remodeling induced by fibroblasts (cell body in yellow) are shown incubated with a culture medium (negative control), b ufPL, c ufP90, and $\mathbf{d}$ CCB. Scale bars: $30 \mu \mathrm{m}$. e Analysis of the spread of fibril orientation of the collagen fibrils $(\kappa)$ as quantification of the collagen organization around the force poles of the embedded cells under the various conditions. Data are represented as means \pm standard deviation (SD) $(n=4)$. Statistically different from control ${ }^{*} p<0.005 ;{ }^{* *} p<0.0005$

ROS assays under abiotic (absence of cells) and biotic (presence of cells) conditions were executed.

The inherent oxidative ability of the different CDPs in abiotic conditions was measured using a DTT assay. All CDPs oxidized DTT in a dose-dependent manner (Fig. 3a). In addition, the abiotic ROS production for all concentrations was significantly different from the control condition.

To further explore the role of oxidative stress under biotic conditions, a DCF-DA staining was performed. In the presence of a wide variety of ROS, the non-fluorescent cell-permeant dye is converted to highly fluorescent cell-retained species. The percentage of fluorescent-positive cells was used for assessing the extent of ROS production in human lung fibroblasts. The analysis showed significant increase in ROS production in cells after $24 \mathrm{~h}$ incubation at even the lowest $\mathrm{CB}$ concentration (Fig. 3b). Moreover, at least eightfold ROS generation compared to the control is detected. No clear dosedependent response curves are observed for the ultrafine particles, in contrast the ROS production stagnates or even declines at higher concentrations.

Contradictory to our results under abiotic conditions, the intracellular ROS production significantly varies between CDP types $(\mathrm{p}<0.05)$. Also, the ROS analysis elucidated similar results for both types of ultrafine particles, while the larger, fine particles showed less ROS production.

\section{Mitochondrial organization and functioning}

First, the organization of the cellular mitochondria was evaluated using CellLight ${ }^{\circledR}$ Mitochondria-GFP expressing fluorescent fusion proteins specifically targeting these organelles. The control cells (Fig. 4a) depicted the typical tubular-like organization indicating healthy mitochondria. In contrast, when the human lung fibroblasts were exposed to CBs (Fig. 4b-d) a substantial loss of the normal morphology of the mitochondrial organization occurred; they became more intermediate (tubular with swelling regions) and even fragmented (globular fragments).

Second, deposition of $\mathrm{CBs}$ in mitochondria was evaluated. The inset of Fig. $4 \mathrm{~b}$ and the orthogonal projection from a z-stack throughout the cell depicted in Additional file 1: Figure S4A clearly show co-localization (indicated by arrowheads and/or yellow color) of the carbonaceous particles, visualized by probing their white-light generation as reported previously [13], and the mitochondria. The colocalization was further quantified by determining the Mander's overlap coefficient (Additional file 1: Figure S4B). The ufPL particulates show an overlap with the mitochondria of $14 \pm 11 \%$. Also, the ufP90 particles show some co-localization with the mitochondrial organization but to a smaller extent ( $9 \pm 8 \%$ Manders' coefficient). In contrast, the larger, fine CCB particles do not show any co-localization with the cellular mitochondria.

Third, we examined the mitochondrial membrane potential (MMP) of the lung fibroblasts in a time course study using MitoTracker ${ }^{\circledR}$ Red CMXROS (Fig. 4e). CMXROS is a fluorochrome that passively diffuses through the membrane of viable cells and is selectively 


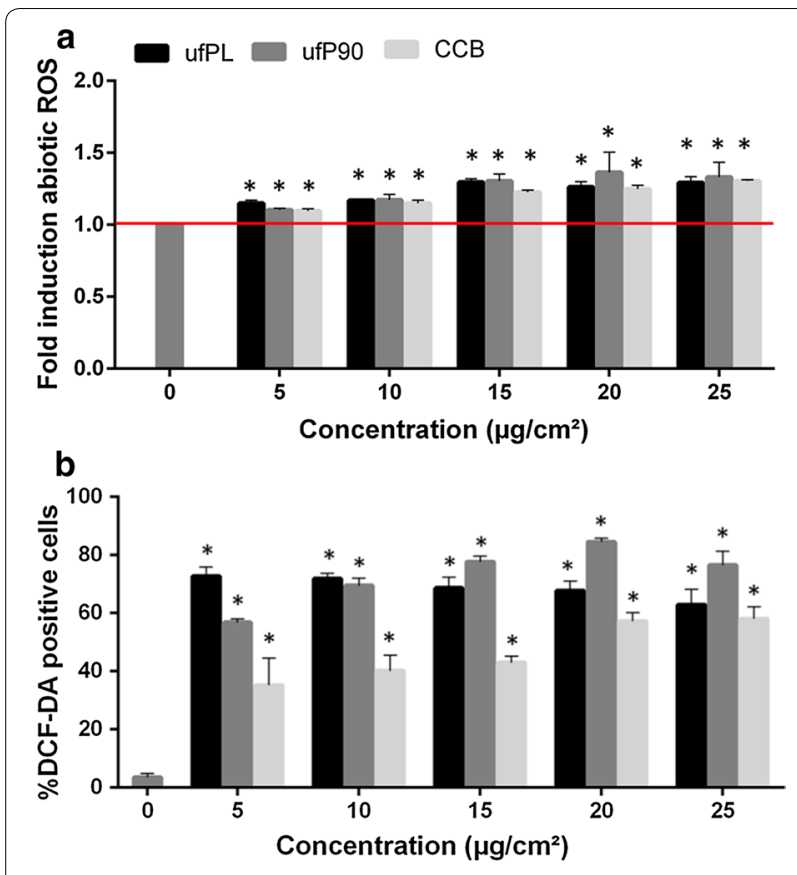

Fig. 3 ROS production of CDPs under abiotic and biotic conditions. a Inherent oxidative potential of the three different CDPs at the various concentrations tested in this study. Red line: the background oxidative level in blank cell culture (IMDM) solution was set as reference. Data are represented as means $\pm S D(n=3)$. * Statistically different from control $p<0.05$. b Human lung fibroblasts (MRC-5 cell line) were exposed to different concentrations $\left(5-25 \mu \mathrm{g} / \mathrm{cm}^{2}\right)$ of three different types of $\mathrm{CBs}$ for $24 \mathrm{~h}$ at $37^{\circ} \mathrm{C}$. At the end of the exposure a DCF-DA assay was conducted to determine ROS production under biotic conditions. All data are represented as means $\pm S D(n=3)$. * Statistically different from control $p<0.0001$

sequestered in mitochondria with an active membrane potential. The study revealed a time-dependent decline of MMP when cells are exposed to CDPs compared to the control, which is significant within 30 or $60 \mathrm{~min}$ for the smallest and largest particles, respectively. In general, the maximum loss of MMP was reached after about $2 \mathrm{~h}$ of exposure. At this point, almost all cells incubated with ufPL and ufP90 lost their MMP, while $20 \%$ of the cells exposed to CCB remained unaffected.

\section{ATP production}

The effect of CDP particles on the cellular adenosine triphosphate (ATP) level was evaluated employing a Cell-Titer glow luminescent cell viability assay. It is clear from the corresponding results (Fig. 5) that the metabolic activity-expressed by the depletion of ATP content-is affected significantly. Additionally, it is observed that the drop in the ATP level of cells treated with the ultrafine ufPL and ufP90 particles is more pronounced than fibroblasts incubated with the larger, fine CCB particulates.

\section{Antioxidants}

To test if the observed toxicological effects are ROSmediated, antioxidants were added to the culture medium of CDP exposed cells. Again, the metabolic activity expressed by the ATP content of the cells is measured after antioxidant treatment.

First, various concentrations of three different antioxidants, $N$-acetyl-L-cysteine (NAC), L-ascorbic acid (vita$\min C$ ) and $\alpha$-tocopherol (vitamin $E$ ), were added to $C C B$ exposed cells. The results show (Fig. 6a) that some specific concentrations of NAC and vitamin E significantly enhance the ATP content of the cells while vitamin C consistently increases the metabolic level from $79 \pm 1 \%$ to $94 \pm 3 \%$.

Next, the concentration-dependent experiment was repeated wherein vitamin $C$, which showed the best and most consistent results in the previous experiment, was added to cells exposed to the ultrafine particulates under study. The corresponding results (Fig. 6b) indicate again a significant improvement of the ATP content of the cells from $55 \pm 3 \%$ to $87 \pm 9 \%$ for ufPL and $37 \pm 3 \%$ to $65 \pm 10 \%$ for ufP90.

Furthermore, the CDP exposed cells' ability to remodel their surrounding matrix was tested after antioxidant treatment with vitamin $C$. The results depicted in Additional file 1: Figure S5 clearly display that under all conditions the collagen fibrils near the force poles of the cells are aligned and thus remodeled. No clear difference is visible anymore between the control cells and CDP exposed cells or between cells exposed to fine or ultrafine particles. This is in agreement with the results on the ATP content of the cells.

\section{Discussion}

\section{CBs characterization}

The CDP types employed in this study are commercial CB. CB is regularly synthesized and used in the global industry market, where employers are exposed to concentrations as high as $650 \mu \mathrm{g} / \mathrm{cm}^{3}$ [16]. In addition, the particles are representative for environmental CDPs to which humans are typically exposed and are often employed as a simplified model for soot or black carbon [17]. A major challenge is the use of relevant particle concentrations, since in vivo exposure cannot be converted directly to in vitro concentrations $[18,19]$. To date, the majority of studies use unrealistically high particle concentrations despite the fact that a few studies have attempted to relate real-life particulate exposure to in vitro concentrations $[18,20]$. These studies have shown that biologically relevant administered concentrations range between 0.2 and $20 \mu \mathrm{g} / \mathrm{cm}^{2}$. Yet, for occupational settings it is known that exposure concentrations can be much higher. 

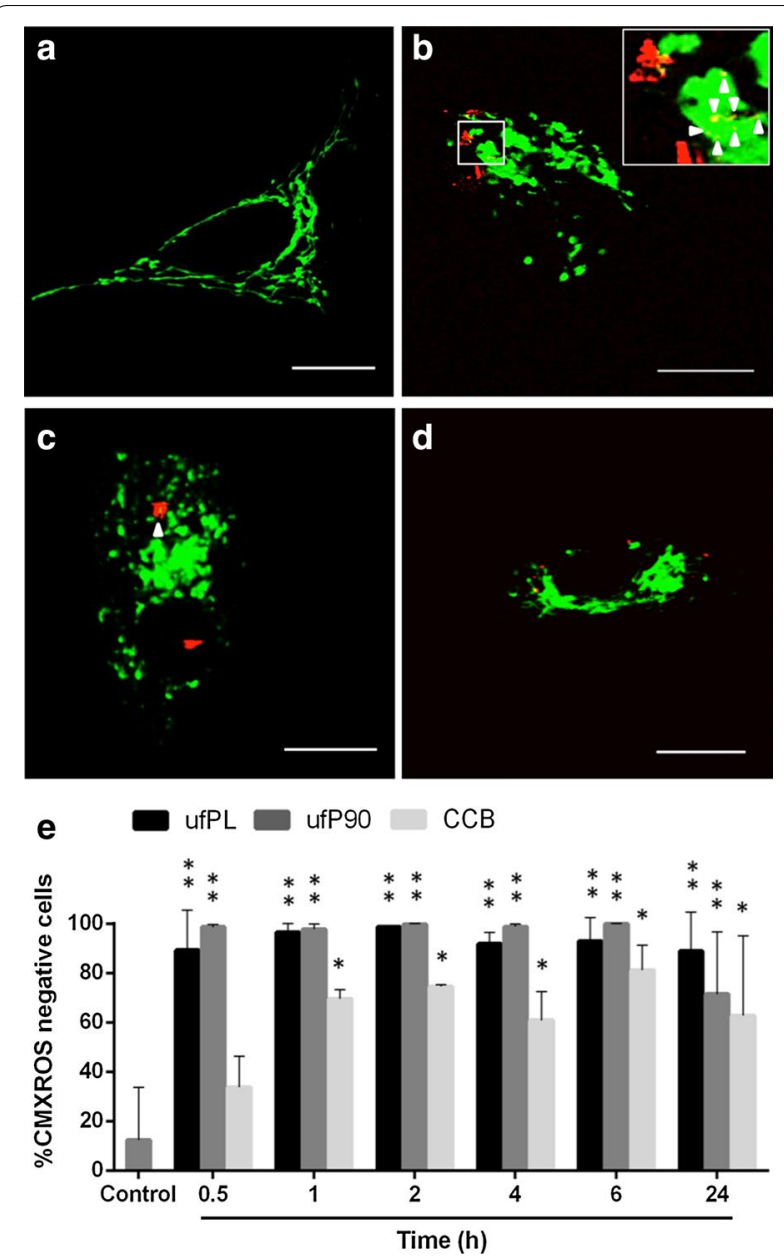

Fig. 4 Mitochondrial damage by CBs in human lung fibroblasts. a-d Human lung fibroblasts (MRC-5 cell line) were exposed for $24 \mathrm{~h}$ to $20 \mathrm{\mu g} / \mathrm{cm}^{2}$ of three different types of CBs at $37^{\circ} \mathrm{C}$. Their mitochondrial organization was examined using CellLight ${ }^{\circledR}$ Mitochondria-GFP (green, Ex/Em 488/510 nm, $3 \mu \mathrm{W}$ radiant power at the samples) and CB particles were imaged under femtosecond pulsed illumination (red, $4 \mathrm{~mW}$ average laser power at the samples, emission detection: 400-410 nm in non-descanned mode). Co-localization between CBs and mitochondria is yellow due to the overlapping colors and additionally indicated by arrowheads. Representative images are shown from: a control condition $\left(0 \mu \mathrm{g} / \mathrm{cm}^{2}\right)$, scale bar: $15 \mu \mathrm{m} ; \mathbf{b}$ incubation with ufPL particles, scale bar: $5 \mu \mathrm{m}$; c incubation with ufP90 particles, scale bar: $5 \mu \mathrm{m}$; d incubation with CCB particles, scale bar: $10 \mu \mathrm{m}$. e Time course study (0.5-24 h) of the loss of mitochondrial membrane potential after exposure to $20 \mathrm{\mu g} / \mathrm{cm}^{2}$ of three different types of $\mathrm{CBs}$ at $37^{\circ} \mathrm{C}$. After incubation, the cells were labeled with CMXROS fluorochromes and the percentage of CMXROS negative cells was determined. Data are represented as means \pm SD $(n=3)$. Statistically different from control marked by $*(p<0.05)$ and $* *(p<0.0005)$

Before cellular exposure, the aggregation state of the CDP particles in aqueous environments was evaluated. All particle types tend to aggregate when suspended in ultrapure water or cell culture medium, which is clear from the TEM images [13] and measured hydrodynamic diameters on samples prepared from aqueous suspensions (Additional file 1: Table S1). This observation is in line with the literature showing similar particle aggregation in cell culture medium [21, 22]. Nevertheless, it has also been reported that aciniform aggregates can maintain the large surface area and other characteristics related to individual (ultra)fine particles [23, 24].

Endotoxins may have adverse effects that can mask the true biological effects of particles if their presence is overlooked [25]. While carbonaceous particles are naturally present in the atmosphere containing all sorts of contaminants, it is still important to have an idea about the possible contribution of endotoxins to observed toxicological effects. The performed LAL assay showed only for one of the three tested CDPs, CCB, a minimal endotoxin level. Note that this small endotoxin quantity is comparable and even lower than generally found in cell culture media and additives such as fetal bovine serum [26]. Additionally, the endotoxin level is lower than the current FDA limit $(0.5 \mathrm{EU} / \mathrm{mL})$ [27] and will not affect the cell culture studies [26].

\section{Cytoskeletal integrity}

In a previous study, we have shown that CDP particles are capable of disturbing the tubulin cytoskeletal structure in human lung fibroblasts [13]. This observation warranted further in depth investigation of the actin cytoskeleton (Additional file 1: Figure S1). Also this cytoskeletal structure appeared to be affected by the presence of the CDP particles in a size-dependent way. Hence, we hypothesized that the cells become mechanically dysfunctional due to the cytoskeletal disturbances after CDP exposure, leading to impaired remodeling of the surrounding matrix.

\section{D cell-induced displacements and matrix remodeling}

To test the hypothesis in a physiological relevant environment, human lung fibroblasts were embedded in a 3D collagen type I hydrogel. Under normal conditions, fibroblasts are able to remodel their surrounding matrix by attaching to collagen fibrils and contract them by exerting mechanical tension [28]. To date, collagen contraction assays are performed to evaluate matrix remodeling [29]. However, results from this type of assay lack crucial information such as changes in the interplay at ECMcellular level and, moreover, the general result is biased by the proliferation rate of the embedded cells. Here, we studied cell-mediated matrix remodeling at the cellular level under normal and exposed conditions using 3D CDM [30]. As far as we know, CDM is employed for the first time to study toxicological effects of (nano)particles. 


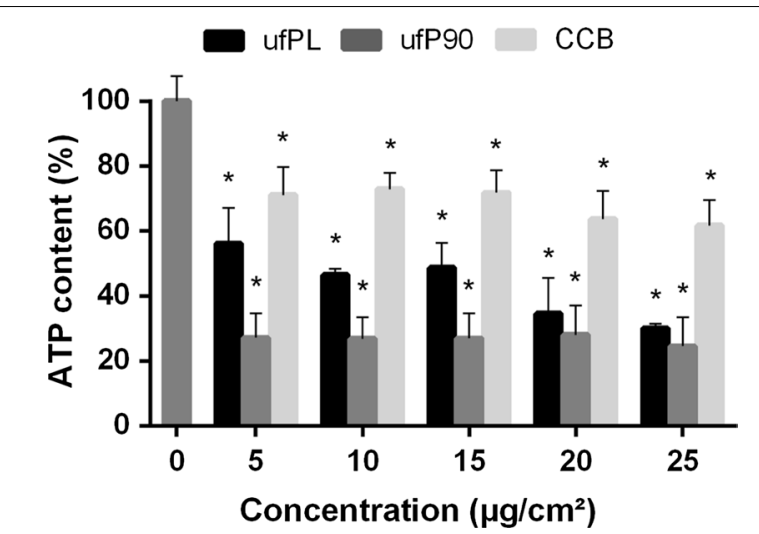

Fig. 5 Intracellular ATP depletion by CBs in human lung fibroblasts. Human lung fibroblasts (MRC-5 cell line) were exposed to different concentrations $\left(5-25 \mu \mathrm{g} / \mathrm{cm}^{2}\right)$ of three different types of CBs for $24 \mathrm{~h}$ at $37^{\circ} \mathrm{C}$. Intracellular ATP content was determined by Cell-Titer glow luminescent cell viability assay. Data are represented as means \pm SD $(n=3) .{ }^{*}$ Statistically different from control $p<0.0001$

3D CDM turned out to be a powerful technique for the evaluation of the mechanical functioning of cells after particulate exposure. The method revealed at the individual cell-level that normal cell-induced displacements are heavily disturbed when cells are incubated with carbonaceous particles (Fig. 1). The label-free second harmonic imaging of the surrounding collagen matrix additionally strengthened this finding. The ECM remodeling around cells incubated with carbon-based particles is much less than the remodeling by control cells (Fig. 2). Moreover, both findings were size-dependent in which the smallest (ufPL and ufP90) particles inhibited the normal cell functioning and corresponding matrix remodeling more than their larger counterparts (CCB). The observed sizedependent effects are of significant relevance since especially the ultrafine particles can penetrate deep into the lung tissue where fibroblasts are residing. Moreover, it is certainly worrying that those smallest particulates have the ability to inhibit fibroblast-mediated matrix remodeling. It is in fact this cell type that has the responsibility to dynamically remodel the ECM during reparative processes in injured or diseased lungs.

A plausible explanation for these findings comprises impaired cell viability. Hence, the extent and mode of cell death was investigated (Additional file 1: Figure S3). Indeed, cells exposed to ultrafine but not fine particles show significant apoptotic death. Yet, inhibition of cellular displacements and matrix remodeling were also seen when cells are incubated with fine particles and moreover only local changes in the vicinity of viable (CellTracker positive) cells were analyzed. Hence, cell viability cannot explain the observed inhibition in matrix displacements and remodeling.

\section{ROS generation}

Earlier literature reports have emphasized the pivotal role of oxidative stress in carbon-based particle toxicology $[11,31]$.

To check oxidative stress as a decisive toxicological factor, as a first step, the inherent oxidative ability of the different CBs in abiotic conditions was measured using a DTT assay.

Interestingly, all particles have similar oxidative potential (Fig. 3a). This is in contrast to literature where sizedependent effects are described [21, 32]. However, it should be noticed that only marginal differences of several nanomols in DTT consumption are reported when comparing different particle sizes and particle concentrations up to $100 \mu \mathrm{g} / \mathrm{cm}^{2}$ are used, which is fourfold higher than those employed in this study [32]. Additionally, the duration of the particles and DTT interaction is not always indicated. Here, we used the same procedure as for CB suspension preparation and cell experiments (for experimental details see "Methods" section), since this will give the most representative results.

Second, the role of oxidative stress under biotic conditions was studied and significant increase in cellular ROS production was observed, even at the lowest $\mathrm{CB}$ concentration (Fig. 3b). The vast majority of ROS is produced under biotic conditions, since at least eightfold ROS generation compared to the control is detected while only a maximum of 1.5 -fold increase was found in abiotic condition. In agreement with earlier reports, no clear dose-dependent response curves are observed; the ROS production stagnates or even declines at higher doses [33, 34]. We hypothesize that the activation of a cellular antioxidant network, which counterbalances ROS at higher concentrations of $\mathrm{CB}$, is responsible for this observation $[35,36]$.

Interestingly, while the particles did not exhibit differential inherent oxidative potential in the absence of cells, their intracellular ROS production significantly varies $(\mathrm{p}<0.05)$. This difference in abiotic and biotic ROS production might be explained by the dissimilar amounts of internalized CBs (as observed by confocal microscopy, see Fig. 4a-d) or diverse interactions of the particulates with their biological target [21], which clearly indicates the need of multi-parametric analysis. Moreover, in line with the expectations, the analysis showed similar results for both ultrafine particles, while the ROS production of the fine particles was less.

\section{Interconnecting ROS production, mitochondrial damage and ATP depletion}

Diverse (nano)particles varying in size and chemical composition have shown to induce structural damage and contribute to oxidative stress in mitochondria [22, 


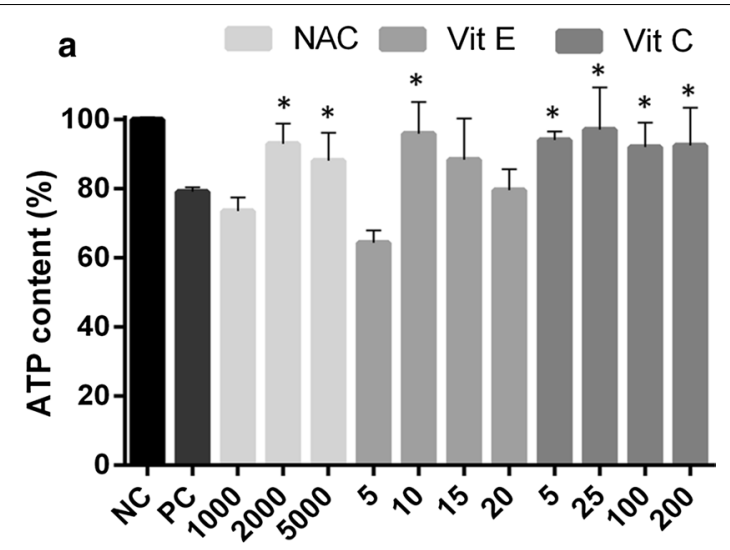

Concentration $(\mu \mathrm{M})$

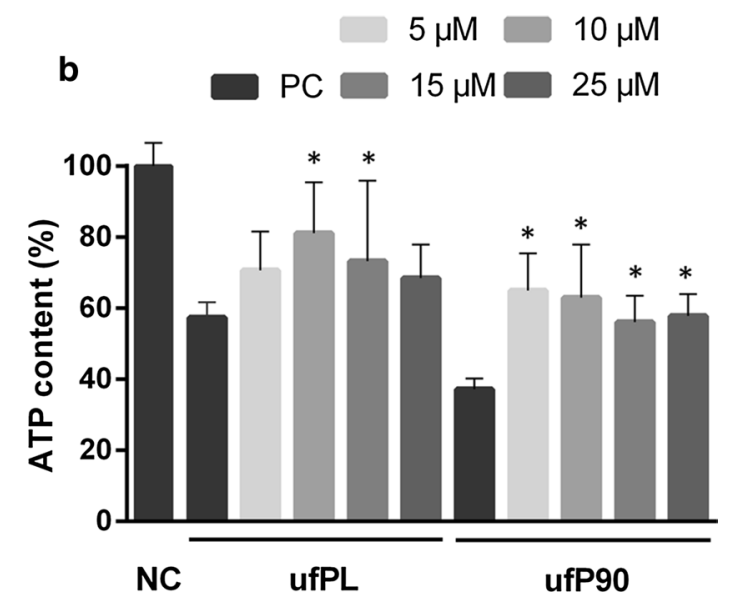

Fig. 6 Improved ATP content in human lung fibroblasts exposed to CDPs and antioxidants. Human lung fibroblasts (MRC-5 cell line) were exposed to $\mathbf{a}$ fine particles or $\mathbf{b}$ ultrafine particles at a concentration of $20 \mathrm{\mu g} / \mathrm{cm}^{2}$ for $24 \mathrm{~h}$ at $37^{\circ} \mathrm{C}$. Next, a three different antioxidants (NAC, Vit. C and Vit. E for 2 h, 2 times $3 \mathrm{~h}$ and 24 h, respectively) or $\mathbf{b}$ Vit. $C$ were added using the concentrations indicated in the graphs. Intracellular ATP content was determined by Cell-Titer glow luminescent cell viability assay. Positive control (PC), corresponding fine or ultrafine particles were added and negative control (NC) plain, complete culture medium was added. Data are represented as means $\pm S D(n=5)$. ${ }^{*}$ Statistically different from control $p<0.05$

35]. Hence, the observed ROS production warrants further investigation of mitochondrial involvement in the toxicological outcome of CDPs.

First, the morphology and integrity of the mitochondria of the cells was evaluated. Mitochondria are morphologically dynamic organelles, which continuously undergo fission and fusion processes to form interconnecting tubular networks into small isolated organelles and vice versa [37]. This enables the cell to meet its metabolic needs and cope with internal or external stresses [38]. The control cells (Fig. 4a) showed the typical tubular morphology indicating healthy mitochondria. In contrast, substantial loss of the common morphology of the mitochondrial organization occurred when human lung fibroblasts were CDP exposed (Fig. 4b-d). The organelles became intermediate as in tubular with swelling regions and even fragmented [39]. This loss of the typical tubular morphology and resulting fragmentation is a strong demonstration of compromised mitochondrial dynamics. In fact, defects in the fission-fusion equilibrium will result in mitochondria that appear swollen and spherical, instead of tubular-like [40]. Overall, this outcome indicates that exposure to CDPs results in mitochondrial structural damage.

Deposition of CDPs in mitochondria may be the reason for mitochondrial damage. Li et al. [41] have already confirmed by employing electron microscopy that ultrafine particles preferentially localized in mitochondria, induce major structural damage and can contribute to oxidative stress. This is in agreement with our current findings. The microscopic images in Fig. 4 and Additional file 1: Figure S4 clearly show co-localization of the mitochondria with the ultrafine (ufPL and ufP90) but not fine (CCB) particles. This can explain the smaller extent of ROS production observed from the latter.

ROS production and subsequent oxidative stress may result in mitochondrial dysfunction. Mitochondria are the major sites of ROS production in mammalian cells [42]. During oxidative phosphorylation, oxygen is reduced to water through controlled addition of electrons via the respiratory chain. However, occasionally some of these electrons escape from the respiratory chain. Electron acceptance by molecular oxygen results in the formation of a range of ROS species such as superoxide anion radicals, hydrogen peroxides and hydroxyl radicals [43]. CDPs localized inside the mitochondria can alter their normal functioning and disrupt the electron transport chain due to either blocking electron transport or accepting an electron and transferring it to molecular oxygen [44]. The maintenance of the MMP in mitochondria is vital for proper oxidative phosphorylation functioning and is considered a critical marker to evaluate mitochondrial perturbation [45, 46]. In agreement with other studies, we found a time-dependent decline of MMP when cells are exposed to CDPs [47]. As loss in MMP alters normal functioning of the electron transport chain, this will ultimately result in enhanced ROS production in mitochondria leading to further mitochondrial membrane damage [45].

ATP is generated by oxidative phosphorylation in mitochondria [48]. As shown by the loss of MMP, damage is caused to the mitochondrial respiratory chain. Figure 5 clearly shows that the metabolic activity-expressed by the depletion of ATP content-is affected significantly when the cells are incubation with CDPs and this in a 
size-dependent manner. This is in agreement with the abovementioned results of mitochondrial damage.

Furthermore, the discussed interconnection between the ROS produced by the CDPs and the resulting cellular ATP depletion is additionally backed by the performed antioxidant experiments (Fig. 6). These experiments show that especially vitamin $\mathrm{C}$ can almost completely restore the ATP content in CCB and ufPL exposed cells and double the ATP level in ufP90 exposed cells. The latter is clearly indicative that the observed cellular toxicological effects are mainly driven by an oxidant-dependent mechanism elucidated by the CDPs. On the other hand, it also suggests that ROS scavenging, antioxidant treatments can restore these detrimental effects.

\section{ATP depletion as plausible inhibitory mechanism of cell-mediated matrix remodeling?}

The key question that remains is how the CDP-induced toxicological effects relate to the impaired matrix displacements. We postulate that the carbon-induced metabolic arrest in the cells, expressed by ATP depletion, is a valid explanation for the disturbed cytoskeleton network and consequently the inhibited ECM remodeling. It is already known that ATP depletion in mammalian cells results in dramatic perturbation of the cytoskeleton. In 1980, Bershadsky et al. [49] already observed that inhibitors of the energy metabolism cause gradual disorganization of actin microfilament bundles in fibroblasts. Additionally, an almost identical mechanism of ATP-dependent cytoskeletal disruption after mitochondrial dysfunction in endothelial cells during simulated ischemia has been described [50]. Summarized, ATP is crucial for the polymerization of the actin component of the cytoskeleton and is therefore required to maintain its structural integrity [51]. The actin network regulates cell shape and the distribution of stresses on the substrate, thereby mediating the mechanical interactions of the cell with the ECM [52-54]. Consequently, if the network is disturbed this may lead to impaired force generation. Note, while this proposed mechanism seems to be contradicted by the inverse difference between the smallest particles (ufPL and ufP90) seen for the ROS and ATP assays on the one hand and cellular displacements on the other hand, only significant differences $(\mathrm{p}<0.05)$ between both particles could be observed for the ROS assay. Furthermore, there is a strong correlation (Pearson correlation coefficient $=0.87$; Goodness of fit $\mathrm{R}^{2}=0.76$ ) between the observations made for mitochondrial dysfunction and the impaired matrix displacements strengthening our postulated hypothesis. Even stronger evidence includes the imaging of the collagen fibril organization remodeled by CDP exposed cells after antioxidant treatment (Additional file 1: Figure S5). While CDP exposed cells lose their ability to remodel their surrounding matrix, they regain their remodeling potential after antioxidant treatment. Hence, the restoration of the cellular ATP level is reflected in recovery of the matrix remodeling by those cells. In summary, the proposed toxicological mechanism of action of the CDPs in the MRC- 5 cells is summarized in Additional file 1: Figure S6.

\section{Conclusions}

Due to the inevitable exposure of humans to polluting combustion-derived particles it is imperative to investigate their potential detrimental effects on human lung cells. In the present study we have proven that carbonaceous particles induce various deleterious effects via an oxidant-dependent mechanism, including: (i) ROS production, (ii) mitochondrial damage and dysfunction, and (iii) ATP depletion. Furthermore, it was shown that matrix displacements were disturbed when human lung fibroblasts were exposed to $\mathrm{CB}$ particles, resulting in the inhibition of matrix remodeling by these cells. Since ATP is a key component for cell-induced matrix contraction, the described oxidant-dependent toxicological mechanism can be a valid explanation for the observed impaired cell-induced remodeling. Moreover, it can be concluded that ultrafine particles cause more adverse effects in human lung fibroblasts compared to their larger counterparts.

These novel insights are of critical importance, since they show that ultrafine carbonaceous particles have the ability to inhibit the physiological relevant function of fibroblasts, namely their mechanical functionality to remodel the extracellular matrix. Dysfunctional fibroblasts may eventually lead to loss of lung tissue structure and functionality. This provides essential information on how those nanoparticles are destructively involved in e.g. impaired tissue remodeling or repair processes in lung damage and diseases associated to inhalation of particulate matter. It also suggests new potential strategies to attenuate the toxicity induced by carbonaceous particle exposure, like for example ROS scavenging antioxidant treatment.

\section{Methods}

\section{Characterization of $C B$ particles}

Three types of carbon black particles (CBs) were tested: ultrafine (uf) carbon black nanopowder (ufPL; PlasmaChem GmbH, Germany), ultrafine Printex 90 (ufP90; Orion Engineered Carbons, Germany) and conductive carbon black nanopowder (CCB; US Research Nanomaterials, USA). Detailed particle characterization was performed in our previous study [13]. For the convenience of the reader, we summarized the main characteristics in Additional file 1: Table S1. 


\section{Cell culture}

Human lung fibroblasts (MRC-5 cell line, ATCC CCL171, LGC Standards, France) were cultured $\left(37{ }^{\circ} \mathrm{C}, 5 \%\right.$ $\mathrm{CO}_{2}$ ) in Minimum Essential Medium (MEM) supplemented with $10 \%$ fetal bovine serum (FBS; Biochrom AG, Germany), $100 \mathrm{U} / \mathrm{mL}$ penicillin, and $100 \mu \mathrm{g} / \mathrm{mL}$ streptomycin.

\section{Confocal imaging}

All imaging was performed using a Zeiss LSM510 META NLO scan head mounted on an inverted laser-scanning microscope (Zeiss Axiovert $200 \mathrm{M}$; Zeiss, Germany) equipped with a $40 \times / 1.1$ water immersion objective.

\section{Cell-induced matrix displacements imaging and quantification}

The effect of $\mathrm{CB}$ particles on the displacements generated by cells was evaluated using cell-induced displacement microscopy according to a method described by us previously [30].

\section{Collagen type I matrix remodeling imaging}

Second harmonic imaging of the fibrils of contracted hydrogels and acquisition of the cell body was done as in the Additional file 1.

\section{General toxicological assays}

The materials and methods of the following assays can be found in the Additional file 1: cell death analysis, abiotic ROS generation, biotic ROS generation, mitochondrial organization and colocalization imaging, mitochondrial functioning assay, metabolic activity assay and antioxidant treatment testing.

\section{Statistical analysis}

In general, every experiment was repeated three times with triplicates of each condition. Data are represented as mean \pm standard deviation and were analyzed using the commercially available software GRAPHPAD (Graphpad Prism 6, Graphpad Software Inc., USA) and JMP (JMP Pro 12, SAS Institute Inc., USA). Analysis of variance (ANOVA) or linear mixed model followed by the posttest Dunnett, for multiple comparisons, were performed.

\section{Additional file}

Additional file 1. Detailed materials and methods and additional figures.

\section{Abbreviations}

2D: two-dimensional; 3D: three-dimensional; ANOVA: analysis of variance; ATP: adenosine triphosphate; BC: black carbon; BSA: bovine serum albumin; CB: carbon black; CCB: conductive carbon black particles; CDPs: combustionderived particles; DCF-DA: 2',7'-dichlorodihydrofluorescein diacetate; DTT: dithiothreitol; ECM: extracellular matrix; EU: endotoxin units; FBS: fetal bovine serum; fs: femtosecond; IMDM: Iscove's modified Dulbecco's medium; LAL: limulus amebocyte lysate; MEM: minimum essential medium; MRC-5: human lung fibroblast cell line; NC: negative control; PBS: phosphate buffered saline; ROS: reactive oxygen species; SD: standard deviation; SHG: second harmonic generation; TEM: transmission electron microscopy; ufP90: ultrafine carbon black (Printex 90) particles from Orion Engineered Carbons; ufPL: ultrafine carbon black particles from PlasmaChem.

\section{Authors' contributions}

HB designed and performed all experiments. JD, LR and MP assisted in experimental work. AJP and HVO contributed to the cell-induced displacement microscopy analysis. MR and MA supervised the project. All authors revised the manuscript and agreed on publication. All authors read and approved the final manuscript.

\section{Author details \\ ${ }^{1}$ Biomedical Research Institute, Hasselt University, Agoralaan Building C, Diepenbeek, Belgium. ${ }^{2}$ Centre for Surface Chemistry and Catalysis, KU Leuven, Celestijnenlaan 200F, Louvain, Belgium. ${ }^{3}$ Institute for Materials Research, Hasselt University, Agoralaan Building D, Diepenbeek, Belgium. ${ }^{4}$ Depart- ment of Mechanical Engineering, KU Leuven, Celestijnenlaan 300C, Box 2419, Louvain, Belgium. ${ }^{5}$ Prometheus, div. Skeletal Tissue Engineering, KU Leuven, Louvain, Belgium.}

\section{Acknowledgements}

H.B. gratefully acknowledges the assistance of Mrs. P. Bex. Prof. dr. H.G. Boyen and dr. B. Conings are acknowledged for their support in determining the purity of the carbon black samples.

\section{Competing interests}

The authors declare that they have no competing interests.

\section{Availability of data and materials}

The datasets generated and analyzed during the current study are available from the corresponding author on reasonable request. The additional information can be found online (filename: Additional file 1).

\section{Consent for publication \\ Not applicable.}

Ethics approval and consent to participate

Not applicable.

\section{Funding}

This research was supported by the Interuniversity Attraction Poles Program (P7/05) initiated by the Belgian Science Policy Office. H.B. acknowledges funding from Research Foundation Flanders (Fonds Wetenschappelijk Onderzoek, FWO) for the doctoral fellowship 11ZB115N. The authors also thank FWO for the research Grant G.0821.13. M.R. acknowledges financial support from the KU Leuven Research Fund (C14/15/053) and FWO (AKUL/15/15 - G0H0816N). M.A. thanks the Province of Limburg (Belgium) for the financial support within the tUL IMPULS FASE II program, allowing for the upgrading of the laser source used in this work. H.V.O. acknowledges funding from the European Research Council under the European Union's Seventh Framework Program (FP7/20072013)/ERC Grant Agreement n³08223).

\section{Publisher's Note}

Springer Nature remains neutral with regard to jurisdictional claims in published maps and institutional affiliations.

Received: 16 August 2018 Accepted: 9 October 2018

Published online: 27 October 2018 


\section{References}

1. Donaldson K, Tran L, Jimenez LA, Duffin R, Newby DE, Mills N, MacNee W, Stone V. Combustion-derived nanoparticles: a review of their toxicology following inhalation exposure. Part Fibre Toxicol. 2005;2:10.

2. Miller MR, Raftis JB, Langrish JP, McLean SG, Samutrtai P, Connell SP, Wilson S, Vesey AT, Fokkens PH, Boere AJF. Inhaled nanoparticles accumulate at sites of vascular disease. ACS Nano. 2017;11:4542-52

3. Maher BA, Ahmed IA, Karloukovski V, MacLaren DA, Foulds PG, Allsop D, Mann DM, Torres-Jardón R, Calderon-Garciduenas L. Magnetite pollution nanoparticles in the human brain. Proc Natl Acad Sci. 2016;113:10797-801.

4. Saenen ND, Bové H, Steuwe C, Roeffaers MB, Provost EB, Lefebvre W, Vanpoucke C, Ameloot M, Nawrot TS. Children's urinary environmental carbon load. A novel marker reflecting residential ambient air pollution exposure? Am J Respir Crit Care Med. 2017;196:873-81.

5. Janssen NA, Hoek G, Simic-Lawson M, Fischer P, Van Bree L, Ten Brink $H$, Keuken M, Atkinson RW, Anderson HR, Brunekreef B. Black carbon as an additional indicator of the adverse health effects of airborne particles compared with PM 10 and PM 2.5. Environ Health Perspect. 2011;119:1691.

6. Krzyzanowski M, Kuna-Dibbert B, Schneider J. Health effects of transportrelated air pollution. In: WHO reports. WHO Regional Office Europe; 2005.

7. Mills NL, Miller MR, Lucking AJ, Beveridge J, Flint L, Boere AJF, Fokkens PH, Boon NA, Sandstrom T, Blomberg A. Combustion-derived nanoparticulate induces the adverse vascular effects of diesel exhaust inhalation. Eur Heart J. 2011;32:2660-71.

8. Grahame TJ, Schlesinger RB. Cardiovascular health and particulate vehicular emissions: a critical evaluation of the evidence. Air Qual Atmos Health. 2010;3:3-27.

9. van Tongeren M, Gardiner K, Rossiter C, Beach J, Harber P, Harrington M. Longitudinal analyses of chest radiographs from the European carbon black respiratory morbidity study. Eur Respir J. 2002;20:417-25.

10. Dobrovolskaia MA, Shurin MR, Kagan VE, Shvedova AA. Ins and outs in environmental and occupational safety studies of asthma and engineered nanomaterials. ACS Nano. 2017;11:7565-71.

11. Donaldson K, Hunter A, Poland C, Smith S. Mechanism of action of combustion-derived nanoparticles. In: Purser D, Maynard R, Wakefield J, editors. Toxicology, survival and health hazards of combustion products. London: Royal Society of Chemistry; 2015. p. 361-81.

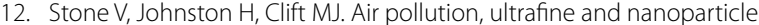
toxicology: cellular and molecular interactions. IEEE Trans Nanobiosci. 2007:6:331-40.

13. Bové H, Steuwe C, Fron E, Slenders E, D’Haen J, Fujita Y, Uji-i H, vandeVen $M$, Roeffaers $M$, Ameloot M. Biocompatible label-free detection of carbon black particles by femtosecond pulsed laser microscopy. Nano Lett. 2016;16:3173-8.

14. White ES. Lung extracellular matrix and fibroblast function. Ann Am Thorac Soc. 2015;12:S30-3.

15. Glaros T, Larsen M, Li L. Macrophages and fibroblasts during inflammation, tissue damage and organ injury. Front Biosci. 2009;14:3988-93.

16. Dai Y, Niu Y, Duan H, Bassig BA, Ye M, Zhang X, Meng T, Bin P, Jia X, Shen M. Effects of occupational exposure to carbon black on peripheral white blood cell counts and lymphocyte subsets. Environ Mol Mutagen. 2016;57:615-22.

17. Poudel BK, Park JH, Lim J, Byeon JH. Direct fluorescent labeling for efficient biological assessment of inhalable particles. Nanotoxicology. 2017;11:953-63.

18. Li N, Hao M, Phalen RF, Hinds WC, Nel AE. Particulate air pollutants and asthma: a paradigm for the role of oxidative stress in PM-induced adverse health effects. Clin Immunol. 2003;109:250-65.

19. Han X, Corson N, Wade-Mercer P, Gelein R, Jiang J, Sahu M, Biswas P, Finkelstein $\mathrm{JN}$, Elder A, Oberdörster $\mathrm{G}$. Assessing the relevance of in vitro studies in nanotoxicology by examining correlations between in vitro and in vivo data. Toxicology. 2012;297:1-9.

20. Phalen RF, Oldham MJ, Nel AE. Tracheobronchial particle dose considerations for in vitro toxicology studies. Toxicol Sci. 2006:92:126-32.

21. Hussain S, Boland S, Baeza-Squiban A, Hamel R, Thomassen LC, Martens JA, Billon-Galland MA, Fleury-Feith J, Moisan F, Pairon J-C. Oxidative stress and proinflammatory effects of carbon black and titanium dioxide nanoparticles: role of particle surface area and internalized amount. Toxicology. 2009;260:142-9.
22. Xia T, Kovochich M, Brant J, Hotze M, Sempf J, Oberley T, Sioutas C, Yeh Jl, Wiesner MR, Nel AE. Comparison of the abilities of ambient and manufactured nanoparticles to induce cellular toxicity according to an oxidative stress paradigm. Nano Lett. 2006;6:1794-807.

23. Long TC, Tajuba J, Sama P, Saleh N, Swartz C, Parker J, Hester S, Lowry GV, Veronesi B. Nanosize titanium dioxide stimulates reactive oxygen species in brain microglia and damages neurons in vitro. Environ Health Perspect. 2007;115:1631-7

24. Phenrat T, Saleh N, Sirk K, Tilton RD, Lowry GV. Aggregation and sedimentation of aqueous nanoscale zerovalent iron dispersions. Environ Sci Technol. 2007:41:284-90.

25. LiY, Boraschi D. Endotoxin contamination: a key element in the interpretation of nanosafety studies. Nanomedicine. 2016;11:269-87.

26. Ryan J. Endotoxins and cell culture. Corning Life Sci Tech Bull. 2004:1-8. http://www.gongyingshi.com/item/doc/20141221/cc_endotoxins _tc_305_rev1.pdf. Accessed 11 Oct 2018.

27. Gorbet MB, Sefton MV. Endotoxin: the uninvited guest. Biomaterials. 2005:26:6811-7.

28. Humphrey JD, Dufresne ER, Schwartz MA. Mechanotransduction and extracellular matrix homeostasis. Nat Rev Mol Cell Biol. 2014;15:802-12.

29. Li B, Wang JHC. Fibroblasts and myofibroblasts in wound healing: force generation and measurement. J Tissue Viability. 2011;20:108-20.

30. Jorge-Penas A, Bové $H$, Sanen K, Vaeyens M-M, Steuwe C, Roeffaers M, Ameloot M, Van Oosterwyck H. 3D full-field quantification of cell-induced large deformation in fibrillary biomaterials by combining non-rigid image registration with label-free second harmonic generation. Biomaterials. 2017:136:86-97.

31. Boland S, Hussain S, Baeza-Squiban A. Carbon black and titanium dioxide nanoparticles induce distinct molecular mechanisms of toxicity. Wiley Interdiscip Rev Nanomed Nanobiotechnol. 2014;6:641-52.

32. Koike $\mathrm{E}$, Kobayashi T. Chemical and biological oxidative effects of carbon black nanoparticles. Chemosphere. 2006;65:946-51.

33. Cao Y, Roursgaard M, Danielsen PH, Møller P, Loft S. Carbon black nanoparticles promote endothelial activation and lipid accumulation in macrophages independently of intracellular ROS production. PLOS ONE. 2014:9:e106711.

34. Frikke-Schmidt H, Roursgaard M, Lykkesfeldt J, Loft S, Nøjgaard JK, Møller P. Effect of vitamin C and iron chelation on diesel exhaust particle and carbon black induced oxidative damage and cell adhesion molecule expression in human endothelial cells. Toxicol Lett. 2011:203:181-9.

35. Manke A, Wang L, Rojanasakul Y. Mechanisms of nanoparticle-induced oxidative stress and toxicity. BioMed Res Int. 2013;2013:942916.

36. Sies H. Oxidative stress. Amsterdam: Elsevier; 2013.

37. Sheridan C, Martin SJ. Mitochondrial fission/fusion dynamics and apoptosis. Mitochondrion. 2010;10:640-8.

38. Youle RJ, Van Der Bliek AM. Mitochondrial fission, fusion, and stress. Science. 2012:337:1062-5.

39. Alaimo A, Gorojod RM, Beauquis J, Munoz MJ, Saravia F, Kotler ML. Deregulation of mitochondria-shaping proteins Opa-1 and Drp-1 in manganese-induced apoptosis. PLoS ONE. 2014;9:e91848.

40. Chen $\mathrm{H}$, Chomyn A, Chan DC. Disruption of fusion results in mitochondrial heterogeneity and dysfunction. J Biol Chem. 2005;280:26185-92.

41. Li N, Sioutas C, Cho A, Schmitz D, Misra C, Sempf J. Ultrafine particulate pollutants induce oxidative stress and mitochondrial damage. Environ Health Perspect. 2003;111:455-60.

42. Murphy MP. How mitochondria produce reactive oxygen species. Biochem J. 2009:417:1-13.

43. Boonstra J, Post JA. Molecular events associated with reactive oxygen species and cell cycle progression in mammalian cells. Gene. 2004;337:1-13.

44. Turrens JF. Mitochondrial formation of reactive oxygen species. J Physiol. 2003;552:335-44

45. Nel A, Xia T, Mädler L, Li N. Toxic potential of materials at the nanolevel. Science. 2006:311:622-7.

46. Santos SM, Dinis AM, Peixoto F, Ferreira L, Jurado AS, Videira RA. Interaction of fullerene nanoparticles with biomembranes: from the partition in lipid membranes to effects on mitochondrial bioenergetics. Toxicol Sci. 2013;138:117-29.

47. Hussain S, Thomassen LC, Ferecatu I, Borot M-C, Andreau K, Martens JA, Fleury J, Baeza-Squiban A, Marano F, Boland S. Carbon black and titanium 
dioxide nanoparticles elicit distinct apoptotic pathways in bronchial epithelial cells. Part Fibre Toxicol. 2010;7:10.

48. Berg JM, Tymoczko JL, Stryer L. Biochemistry. 5th ed. W.H: Freeman; 2002.

49. Bershadsky A. Actin cytoskeleton of spread fibroblasts appears to assemble at the cell edges. J Cell Sci. 1986;82:235-48.

50. Nishimura Y, Romer LH, Lemasters JJ. Mitochondrial dysfunction and cytoskeletal disruption during chemical hypoxia to cultured rat hepatic sinusoidal endothelial cells: the $\mathrm{pH}$ paradox and cytoprotection by glucose, acidotic pH, and glycine. Hepatology. 1998;27:1039-49.

51. Pollard TD, Borisy GG. Cellular motility driven by assembly and disassembly of actin filaments. Cell. 2003;112:453-65.
52. Geiger B, Spatz JP, Bershadsky AD. Environmental sensing through focal adhesions. Nat Rev Mol Cell Biol. 2009;10:21-33.

53. Schwarz US, Gardel ML. United we stand -integrating the actin cytoskeleton and cell-matrix adhesions in cellular mechanotransduction. J Cell Sci. 2012;125:3051-60.

54. Oakes PW, Gardel ML. Stressing the limits of focal adhesion mechanosensitivity. Curr Opin Cell Biol. 2014;30:68-73.
Ready to submit your research? Choose BMC and benefit from:

- fast, convenient online submission

- thorough peer review by experienced researchers in your field

- rapid publication on acceptance

- support for research data, including large and complex data types

- gold Open Access which fosters wider collaboration and increased citations

- maximum visibility for your research: over $100 \mathrm{M}$ website views per year

At BMC, research is always in progress.

Learn more biomedcentral.com/submissions 